International Journal of Pure and Applied Mathematics

Volume 93 No. 4 2014, 541-547

ISSN: 1311-8080 (printed version); ISSN: 1314-3395 (on-line version)

url: http://www.ijpam.eu

doi: http://dx.doi.org/10.12732/ijpam.v93i4.5

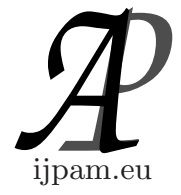

\title{
FEEDBACK NUMBERS OF FLOWER SNARK AND RELATED GRAPHS
}

\author{
Sijia Zhang ${ }^{1}$, Xirong $\mathrm{Xu}^{2}{ }^{\S}$, Cong $\mathrm{Liu}^{3}$, Yuansheng Yang ${ }^{4}$ \\ School of Computer Science and Technology \\ Dalian University of Technology \\ Dalian, 116024, P.R. CHINA
}

\begin{abstract}
A subset of vertices of a graph $G$ is called a feedback vertex set of $G$ if its removal results in an acyclic subgraph. The minimum cardinality of a feedback vertex set is called the feedback number. In this paper, we investigate the feedback number of flower snark and related graphs $H_{n}$. Let $f\left(H_{n}\right)$ denote the feedback number of $H_{n}$, we prove that
\end{abstract}

$$
f\left(H_{n}\right)=n+1 \text { for } n \geq 3 .
$$

AMS Subject Classification: 05C85, 68R10

Key Words: feedback vertex set, feedback number, flower snark, acyclic subgraph, bipartite subgraph

\section{Introduction}

Let $G=(V, E)$ be a graph or digraph without multiple edges, with vertex set $V(G)$ and edge set $E(G)$. A subset $F \subset V(G)$ is called a feedback vertex set if the subgraph $G-F$ is acyclic, that is, if $G-F$ is a forest. The minimum cardinality of a feedback vertex set is called the feedback number (or decycling number proposed first by Beineke and Vandell [1]) of $G$. A feedback vertex set of this cardinality is called a minimum feedback vertex set.

Received: January 7, 2014

(C) 2014 Academic Publications, Ltd.

${ }^{\S}$ Correspondence author url: www.acadpubl.eu 
Apart from its graph-theoretical interest, the minimum feedback vertex set problem has important application to several fields. For example, the problems are in operating systems to resource allocation mechanisms that prevent deadlocks [2], in artificial intelligence to the constraint satisfaction problem and Bayesian inference, in synchronous distributed systems to the study of monopolies and in optical networks to converters placement problem(see [3] and $[4])$.

Determining the feedback number is quite difficult even for some elementary graphs. However, the problem has been studied for some special graphs and digraphs, such as hypercubes, meshes, toroids, butterflies, cube-connected cycles, directed split-stars (see [3]- [13]). In fact, the minimum feedback set problem is known to be $N P$-hard for general graphs [14] and the best known approximation algorithm is one with an approximation ratio two [5].

Many research of literatures have been studied about flower snark and its related graphs. For example, Zheng. [15] has been studied the crossing number of flower snark and its related graph; Xi. [16] has been studied super vertexmagic total labelings of flower snark and related graphs; Mo.hammad [17] and Tong. [18] have been studied labeling of flower snark and related graphs; In addition, the adjacent vertex distinguishing incidence coloring number of flower graphs has been studied in area of mathematics. But, there is little research done so far about the feedback number of flower snark and related graphs.

In this paper, we consider the feedback number of flower snark and related graphs $H_{n}$. Let $f\left(H_{n}\right)$ denote the feedback number of $H_{n}$, we proves that:

$$
f\left(H_{n}\right)=n+1, \text { for } n \geq 3
$$

\section{Feedback Vertex Set of $H_{n}$}

Let $G_{n}$ be a simple nontrivial connected cubic graph with vertex set $V\left(G_{n}\right)=$ $\left\{a_{i}, b_{i}, c_{i}, d_{i}: 0 \leq i \leq n-1\right\}$ and edge set

$$
E\left(G_{n}\right)=\left\{a_{i} a_{i+1}, b_{i} b_{i+1}, c_{i} c_{i+1}, d_{i} a_{i}, d_{i} b_{i}, d_{i} c_{i}: 0 \leq i \leq n-1\right\},
$$

where the vertex labels are read modulo $n$.

Let $H_{n}$ be a graph obtained from $G_{n}$ by replaceing the edges $b_{n-1} b_{0}$ and $c_{n-1} c_{0}$ with $b_{n-1} c_{0}$ and $c_{n-1} b_{0}$ respectively. For odd $n \geq 5, H_{n}$ is called a Snark, namely Flower Snark [19] and [20]. $G_{n}$ and $H_{n}(n=3$ or even $n \geq 4)$ are called the related graphs of Flower Snark [21]. 
In this paper, we denote $H_{n}$ as Flower Snark and its related graphs. By the definition of $H_{n}$, the set of $V\left(H_{n}\right)$ can be divided into three parts as follows:

$$
\left\{\begin{array}{l}
H_{1}=\left\{a_{i} a_{i+1}: 0 \leq i \leq n-1\right\} \\
H_{2}=\left\{b_{j} b_{j+1}, b_{n-1} c_{0}, c_{k} c_{k+1}, c_{n-1} b_{0}: 0 \leq j, k \leq n-2\right\} \\
H_{3}=\left\{d_{i} a_{i}, d_{i} b_{i}, d_{i} c_{i}: 0 \leq i \leq n-1\right\}
\end{array}\right.
$$

Obviously, $H_{1}$ is the only one cycle which contains all the vertices for $\left\{a_{i}\right\}, H_{2}$ is the only one cycle which contains all the vertices for $\left\{b_{j}\right\}$ and $\left\{c_{k}\right\} . H_{1} \cap H_{2}=\emptyset$ and $H_{1} \cup H_{2} \cup H_{3}=H_{n}$.

Denote

$$
\left\{\begin{array}{l}
F_{1}=\left\{a_{n-1}\right\} \\
F_{2}=\left\{c_{n-1}\right\} \\
F_{3}=\left\{d_{i}: 0 \leq i \leq n-2\right\}
\end{array}\right.
$$

and denote $F_{n}=F_{1} \cup F_{2} \cup F_{3}$, we have the following result.

Lemma 1. $F_{n}$ is a feedback vertex set of $H_{n}$ for $n \geq 3$.

Proof. Since $H_{1}$ is a cycle, then we only delete any one vertex, say $a_{n-1}$, to obtain an acyclic subgraph of $H_{1}$ denoted by $G\left[N_{1}\right]$, which implies that $G\left[N_{1}\right]=G\left[H_{1} \backslash F_{1}\right]$.

Similarly, in $H_{2}$ we only delete any one vertex, say $c_{n-1}$, to obtain an acyclic subgraph denoted by $G\left[N_{2}\right]$, which implies that $G\left[N_{2}\right]=G\left[H_{2} \backslash F_{2}\right]$.

Since $H_{1} \cap H_{2}=\emptyset$, then $G\left[N_{1}\right] \cap G\left[N_{2}\right]=\emptyset$, that is , $G\left[N_{1}\right] \cup G\left[N_{2}\right]$ is an acyclic graph.

We denote $G\left[H_{3} \backslash F_{3}\right]$ as $G\left[N_{3}\right]$ which include only one edge $d_{n-1} b_{n-1}$. Obviously, $V\left(G\left[N_{3}\right]\right) \cap V\left(G\left[N_{2}\right]\right)=\left\{b_{n-1}\right\}, V\left(G\left[N_{3}\right]\right) \cap V\left(G\left[N_{1}\right]\right)=\emptyset$. Its relationships are shown as Figure.1.

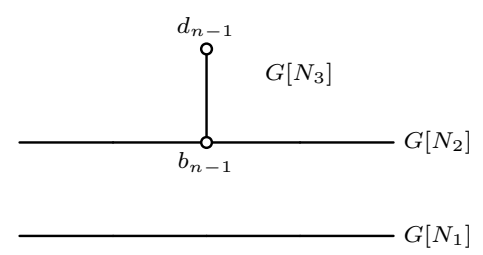

Figure 1: The relationship of $G\left[N_{1}\right], G\left[N_{2}\right]$ and $G\left[N_{3}\right]$.

It is a simple matter to verify that $G\left[H_{n} \backslash\left(F_{1} \cup F_{2} \cup F_{3}\right)\right]$ is an acyclic graph. By the definition of feedback vertex set, $F_{n}$ is a feedback vertex set of $H_{n}$. 


\section{Feedback Number of $H_{n}$}

Lemma 2. For feedback vertex set $F_{n}$ in a graph $G=(V, E)$ with maximum degree $\Delta$, it holds that

$$
F_{n} \geq\left\lceil\frac{|E|-|V|+1}{\Delta-1}\right\rceil
$$

Lemma 3. The feedback vertex set in $H_{n}$ is of size at least:

$$
f\left(H_{n}\right) \geq n+1, \text { for } n \geq 3
$$

Proof. Noting that $H_{n}(n \geq 3)$ has $4 n$ vertices and $6 n$ edges, the maximum degree is 3 , by Lemma 2, we immediately obtain the lower bound

$$
f\left(H_{n}\right) \geq\left\lceil\frac{6 n-4 n+1}{3-1}\right\rceil=\left\lceil\frac{2 n+1}{2}\right\rceil=n+1 .
$$

Theorem 1. Feedback vertex number of flower snark and related graphs $H_{n}$ :

$$
f\left(H_{n}\right)=n+1, \text { for } n \geq 3 .
$$

Proof. For $n \geq 3$, by definition, it is easy to find that $\left|F_{1}\right|=\left|F_{2}\right|=1$, $\left|F_{3}\right|=n-1$.

Since $F_{n}$ is a feedback vertex set, then we have $f\left(H_{n}\right) \leq\left|F_{n}\right|=1+1+n-1=$ $n+1$. In addition, $f\left(H_{n}\right) \geq n+1$, we conclude that

$$
f\left(H_{n}\right)=n+1, \text { for } n \geq 3
$$

The theorem holds.

In Figure 2, we show the feedback vertex sets of several $H_{n}$ graphs with small $n$, where the vertices of feedback vertex sets are in red, the acyclic graph are in blue.

\section{Acknowledgments}

The work was supported by NNSF of China (No.61170303). 

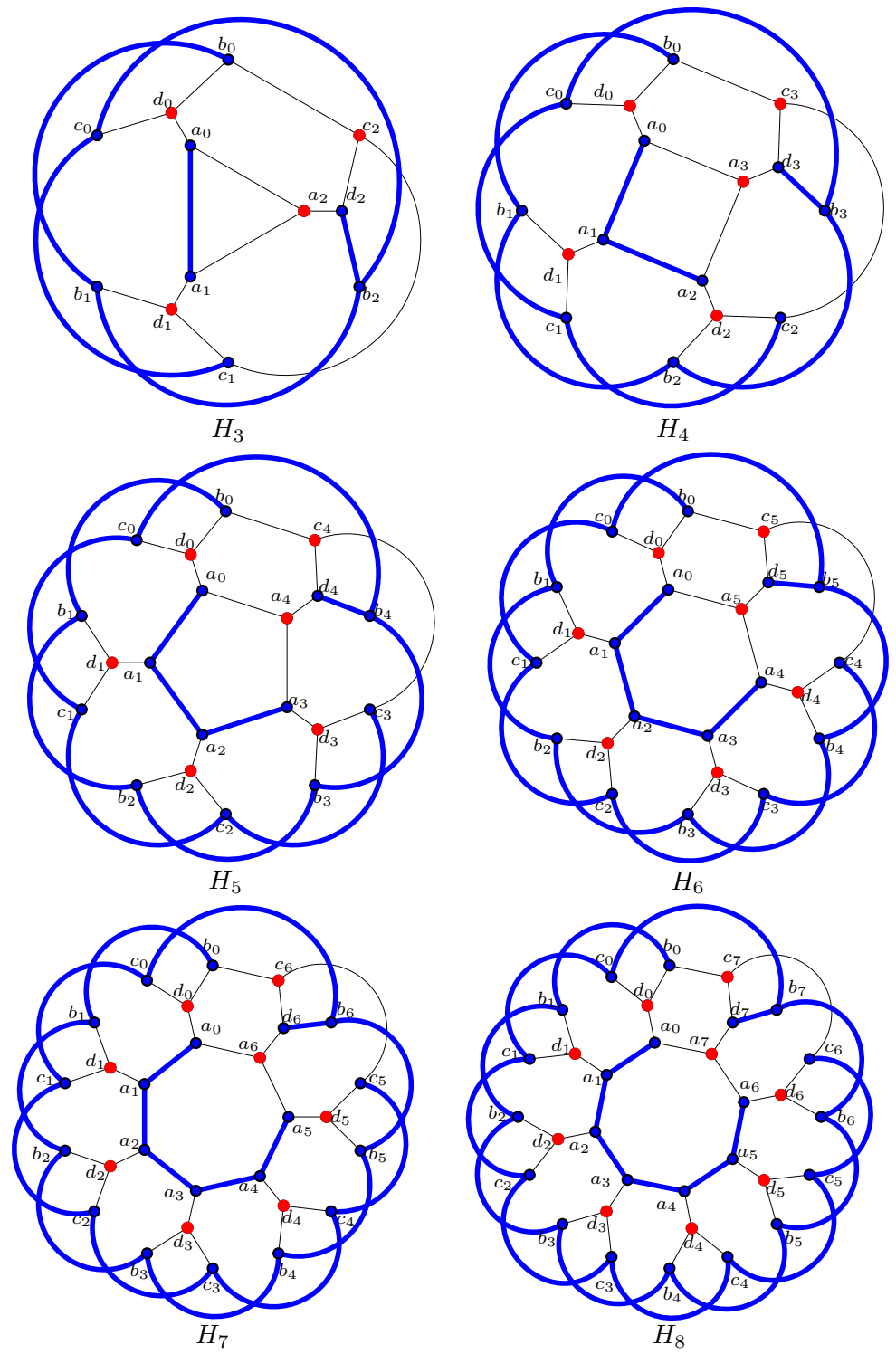

Figure 2: Flower snark and its related graph of $H_{n}$

\section{References}

[1] L. W. Beineke, R. C. Vandell, Decycling graphs, J. Graph Theory., 25, (1997), 59-77. 
[2] I. Niven, H. S. Zuckerman, An Introduction to the Theory of Numbers (5th ed.), John Wiley and Sons, New York, (1991).

[3] I. Caragiannis, C. Kaklamanis, P. Kanellopoulos, New bounds on the size of the minimum feedback vertex set in meshes and butterflies, Information Processing Letters, 83, (2002), 75-80.

[4] P. Festa, P. M. Pardalos, M. G. C. Resende, Feedback set problems, Handbook of Combinatorial Optimization, A, (1999), 209-258.

[5] V. Bafna, P. Berman, T. Fujito, A 2-approximation algorithm for the undirected feedback vertex set problem, SIAM J. Discrete Mathematics, 12, (1999), 289-297.

[6] S. Bau, L. W. Beineke, Z. Liu, G. Du, R. C. Vandell, Decycling cubes and grids, Utilitas Math., 59, (2001), 129-137.

[7] R. Bar-Yehuda, D. Geiger, J. S. Naor, R. M. Roth, Approximation algorithms for the feedback vertex set problem with applications to constraint satisfaction and Bayesian inference, SIAM J. Comput., 27, (1998), 942959.

[8] R. Focardi, F. L. Luccio, D. Peleg, Feedback vertex set in hypercubes, Information Processing Letters, 76, (2000), 1-5.

[9] Y. D. Liang, On the feedback vertex set in permutation graphs, Information Processing Letters, 52, 123-129, (1994).

[10] F. L. Luccio, Almost exact minimum feedback vertex set in meshes and butterflies, Information Processing Letters, 66, (1998), 59-64.

[11] G. W. Smith, Jr. and R. B. Walford, The identification of a minimal feedback vertex set of a directed graph, IEEE Trans. Circuits and Systems, 22, (1975), 9-15.

[12] C.C. Wang, E. L. Lloyd, M. L. Soffa, Feedback vertex sets and cyclically reducible graphs, J. Assoc. Comput. Mach., 32, (1985), 296-313.

[13] F.H. Wang, C.J. Hsu, J.C. Tsai, Minimal feedback vertex sets in directed split-stars, Networks, 45, (2005), 218-223.

[14] M.R.Garey,D.S.Johnson, Computers and Intractability, Freeman, San Francisco, CA, (1979). 
[15] W.P. Zheng, X.H. Lin, Y.S. Yang, The crossing number of flower snarks and related graphs, Ars Combinatoria, 86, (2008), 57-64.

[16] Y. Xi, Y.S. Yang, L.P. Wang, Super Vertex-Magic Total Labelings of Flower Snark and Related Graph. Indian Journal Of Pure Applied Mathematics, Indian Journal of Pure Applied Mathematics , 40(2), (2009), 123-129.

[17] Kh. Md. Mominul, X.H. Lin,Y.S. Yang, L.P. Wang, Prime Cordial labeling of Flower Snark and related graphs, Ars Combinatoria, 105, (2012), 45-52.

[18] C.L. Tong, X.H. Lin,Y.S. Yang, L.P. Wang,L(2,1)-labeling of flower snark and related graphs, Ars Combinatoria, 110,(2013), 505-512.

[19] A.Cavicchioli, M.Meschiari, B.Ruini,and F.Spaggiari. A Survey on Snarks and New Results: Products, Reducibility and a Computer Search, Journal of Graph Theory, 28, (1998),57-86.

[20] Eckhard Steffen. Classifications and characterizations of snarks, Discrete Mathematics, 188,(1998), 183-203.

[21] A.Cavicchioli, T.E.Murgolo, B.Ruini and F.Spaggiari. Special Classes of Snarks, Acta Applicandae Mathematicae, 76,(2003), 57-88. 
